\title{
Dietary Fibre, Gut Microbiota Dysbiosis and Type 2 Diabetes ${ }^{+}$
}

\author{
Omorogieva Ojo ${ }^{1, *}$, Qianqian Feng ${ }^{2}$, Osarhumwese Osaretin Ojo ${ }^{3}$ and Xiaohua Wang ${ }^{2}$ \\ 1 Faculty of Education, Health and Human Sciences, School of Health Sciences, University of Greenwich, \\ Avery Hill Campus, Avery Hill Road, London SE9 2UG, UK \\ 2 The School of Nursing, Soochow University, Suzhou 215006, China; 20195231027@stu.suda.edu.cn (Q.F.); \\ wangxiaohua@suda.edu.cn (X.W.) \\ 3 South London and Maudsley NHS Foundation Trust, University Hospital, Lewisham High Street, \\ London SE13 6LH, UK; Osarhumwese.Ojo@slam.nhs.uk \\ * Correspondence: o.ojo@greenwich.ac.uk; Tel.: +44-20-8331-8626; Fax: +44-20-8331-8060 \\ + Presented at the 1st International Electronic Conference on Nutrients-Nutritional and Microbiota Effects on \\ Chronic Disease, 2-15 November 2020; Available online: https://iecn2020.sciforum.net/
}

Published: 30 October 2020

\begin{abstract}
Background: Diabetes prevalence is on the increase globally and its impact on those with the condition in terms of acute and chronic complications can be profound. People with type 2 diabetes constitute the majority of those with diabetes and the risk factors include obesity, lifestyle and gut microbiota dysbiosis. Poor dietary intake has been reported to influence the community of gut microbiome. Therefore, higher intake of dietary fibre may alter the environment in the gut and promote microbial growth and proliferation. Aim: This is a systematic review and meta-analysis which examined the effect of dietary fibre on gut microbiota in patients with type 2 diabetes. Method: This review was conducted in line with the PRISMA framework. Databases were searched for relevant articles which were screened based on inclusion and exclusion criteria. Results: Nine articles which met the inclusion criteria were selected for the systematic review and meta-analysis. High dietary intake significantly improved $(p<0.05)$ the abundance of Bifidobacterium, total SCFAs and HbA1c. Discussion: The promotion of SCFA producers in greater diversity and abundance by dietary fibre may have resulted in improvement in glycated haemoglobin, partly due to increased GLP-1 production. Conclusion: Consumption of high dietary fibre has significant $(p<0.05)$ effect on Bifidobacterium, total SCFAs and HbA1c, but not $(p>0.05)$ on propionic, butyric, and acetic acid, fasting blood glucose and HOMAR-IR.
\end{abstract}

Keywords: type 2 diabetes; dietary fibre; gut microbiota; dysbiosis; short chain fatty acids; glycated haemoglobin; fasting blood glucose; adverse events

\section{Introduction}

The prevalence of type 2 diabetes is increasing worldwide, both in developed countries and in developing economies [1,2]. Furthermore, type 2 diabetes represents about $90 \%$ of adults who are diagnosed with diabetes, often associated with acute (such as hyperglycaemia, diabetic ketoacidosis, hyperosmolar hyperglycaemic state) and chronic complications (including kidney dysfunction, neuropathy, retinopathy and cardiovascular diseases) [3,4]. A range of factors such as overweight and obesity, lifestyle, genetic predisposition and gut microbiota dysbiosis have been implicated in the aetiology of type 2 diabetes [5]. In particular, low intake of dietary fibre and consumption of foods that are high in fat and sugar which are common in western lifestyle have been reported to contribute to the depletion in the abundance of specific bacteria taxa and the diversity of the gut microbial community [6]. Therefore, it is possible that a higher intake of dietary fibre may alter the environment Proceedings 2020, 4, x; doi: FOR PEER REVIEW www.mdpi.com/journal/proceedings 
in the gut and provide the needed substrate for microbial bloom. Our current understanding of the exact relationships between gut microbiota and diseases is limited and continues to evolve $[7,8]$. While previous reviews have focused on the role of general diet on gut microbiota, the current review is a systematic review and meta-analysis which evaluated the role of dietary fibre in modulating gut microbiota dysbiosis in patients with type 2 diabetes.

\section{Method}

The Preferred Reporting Items for Systematic Reviews and Meta-Analyses (PRISMA) was the framework used for this review [9]. Various electronic databases including EBSCOHost with links to Health Sciences Research Databases (encompassing Academic Search Premier, MEDLINE, Psychology and Behavioral Sciences Collection, APA PsycInfo, CINAHL Plus with Full Text and APA PsycArticles databases), EMBASE and Google Scholar were searched for relevant articles. The reference lists of articles were also searched. Searches were conducted from date of commencement of database to 5 August 2020. The search strategy was based on Population, Intervention, Comparator, Outcomes, Studies (PICOS) framework and involved the use of synonyms and medical subject headings (MesH). Search terms were combined with Boolean operators (OR/AND). Results of the searches were screened for eligibility based on inclusion and exclusion criteria. Only randomised controlled trials were included in the review and the studies were evaluated for quality and risk of bias [10]. The meta-analysis was conducted using Review Manager 5.3 software [11].

\section{Results}

Nine studies which met the inclusion criteria were selected for the systematic review and metaanalysis and four distinct areas were identified: The effect of dietary fibre on gut microbiota; The role of dietary fibre on short chain fatty acids (SCFAs); Glycaemic control and Adverse events. There was significant improvement $(p<0.01)$ in the relative abundance of Bifidobacterium with a mean difference of $0.72(95 \% \mathrm{CI}, 0.56,0.89)$ in the dietary fibre group compared with placebo (Figure 1).

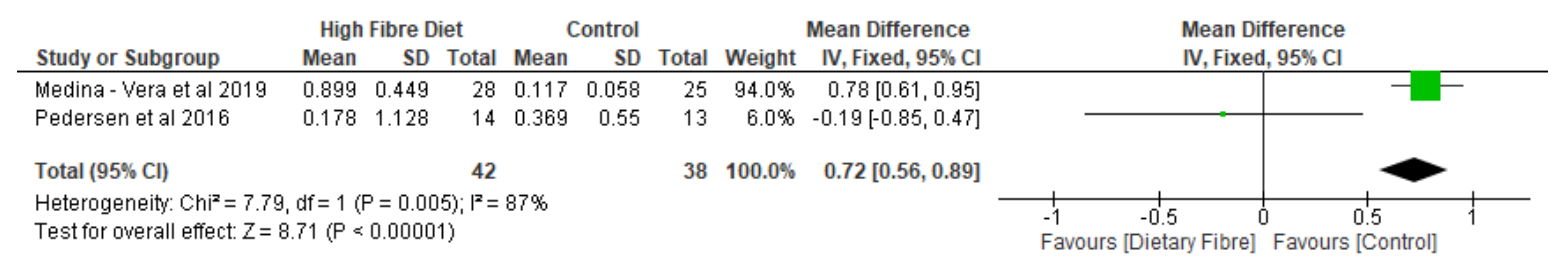

Figure 1. Shows the effect of dietary fibre on Bifidobacterium (\%).

In relation to the meta-analysis for SCFAs, while there was significant difference $(p=0.02)$ between the dietary fibre group and placebo with a standardised mean difference of 0.5 (95\% CI, 0.08 , 0.91 ) regarding total SCFAs (Figure 2), the differences were not significant $(p>0.05)$ in relation to acetic acid, propionic acid and butyric acid.

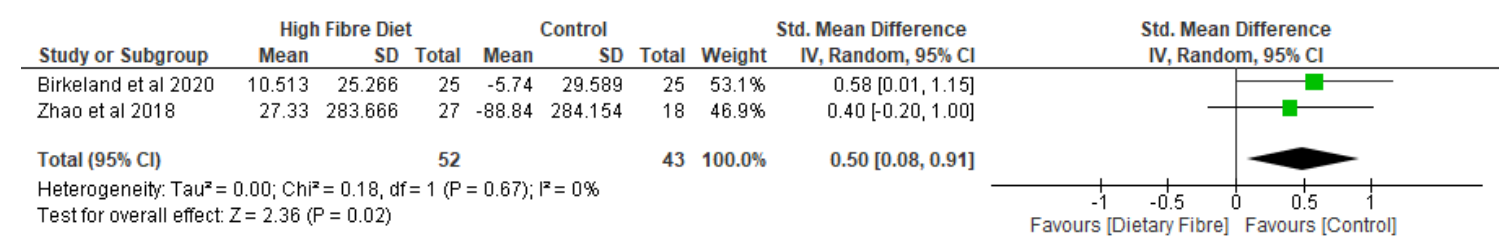

Figure 2. Shows the effect of dietary fibre on Total Short Chain Fatty Acids.

There was only significant improvement $(p=0.002)$ with respect to glycated haemoglobin with a mean difference of $-0.18(95 \% \mathrm{CI},-0.29,-0.06)$ in the dietary fibre group compared with placebo group (Figure 3). Differences between the two groups were not significant $(p>0.05)$ in relation to fasting blood glucose and Homeostatic Model Assessment of Insulin Resistance (HOMAR-IR). 
Furthermore, there were no significant differences between the two groups in subjects who reported adverse events.

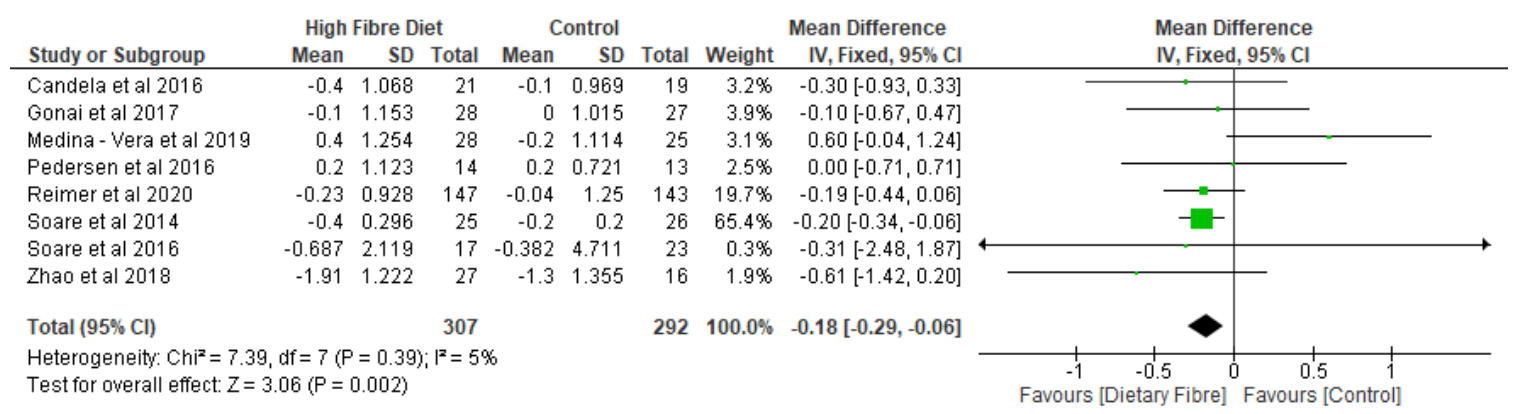

Figure 3. Shows the effect of dietary fibre on Glycated Haemoglobin (\%).

\section{Discussion}

It is possible that the promotion of SCFA producers in greater diversity and abundance by dietary fibre in this review led to improvement in glycated haemoglobin, partly due to increased glucagon-like peptide-1 (GLP-1) production [12]. In addition, Bifidobacterium lactis has been reported to increase glycogen synthesis, decrease expression of hepatic gluconeogenesis-genes, improve translocation of glucose transport- 4 and promote glucose uptake [13]. It is also possible that the reduction in body weight of participants in the intervention group compared with control may have contributed to the observed improvement in glycated haemoglobin in the dietary fibre group [14].

\section{Conclusions}

This systematic review and meta-analysis have demonstrated that dietary fibre can significantly improve $(p<0.05)$ the relative abundance of Bifidobacterium, total SCFAs and glycated haemoglobin. However, dietary fibre did not appear to have significant effect $(p>0.05)$ on fasting blood glucose, HOMAR-IR, acetic acid, propionic acid, butyric acid and adverse events.

Author Contributions: Conceptualization, O.O., O.O.O. and X.W.; methodology, O.O., Q.F., O.O.O. and X.W.; validation, O.O., X.W. and O.O.O.; formal analysis, O.O., Q.F., X.W.; writing-original draft preparation, O.O.; writing -review and editing, O.O., Q.F., O.O.O. and X.W. All authors have read and agreed to the published version of the manuscript.

Funding: This research received no external funding.

Conflicts of Interest: The authors declare no conflict of interest.

\section{References}

1. World Health Organisation. Global Report on Diabetes. 2016. Available online: file://D:/World\%20Health\%20Organisation\%20(WHO)\%20Global\%20Report\%20on\%20Diabetes.pdf (accessed on 01 August 2020).

2. Woldeamlak, B.; Yirdaw, K.; Biadgo, B. Role of Gut Microbiota in Type 2 Diabetes Mellitus and Its Complications: Novel Insights and Potential Intervention Strategies. Korean J. Gastroenterol. 2019, 74, 314320. doi:10.4166/kjg.2019.74.6.314.

3. National Institute for Health and Care Excellence (NICE). Type 2 Diabetes in Adults: Management. 2015. Available online: https://www.nice.org.uk/guidance/ng28 (accessed on 01 August 2020).

4. Ojo, O. An overview of diabetes and its complications. Diabetes Res. Open J. 2016, 2, e4-e6. doi:10.17140/DROJ-2-e005

5. Ebrahimzadeh Leylabadlo, H.; Sanaie, S.; Sadeghpour Heravi, F.; Ahmadian, Z.; Ghotaslou, R. From role of gut microbiota to microbial-based therapies in type 2-diabetes. Infect. Genet. Evol. 2020, 81, doi:10.1016/j.meegid.2020.104268. 
6. Makki, K.; Deehan, E.C.; Walter, J.; Bäckhed, F. The Impact of Dietary Fiber on Gut Microbiota in Host Health and Disease. Cell Host Microbe 2018, 23, 705-715, doi:10.1016/j.chom.2018.05.012.

7. Duvallet, C.; Gibbons, S.M.; Gurry, T.; Irizarry, R.A.; Alm, E.J. Meta-analysis of gut microbiome studies identifies disease-specific and shared responses. Nat. Commun. 2017, 8, 1784, doi:10.1038/s41467-017-019738.

8. Scientific Advisory Committee on Nutrition. Carbohydrate and Health. 2015. Available online: https://assets.publishing.service.gov.uk/government/uploads/system/uploads/attachment_data/file/44550 3/SACN_Carbohydrates_and_Health.pdf (accessed on 16 August 2020).

9. Moher, D.; Liberati, A.; Tetzlaff, J.; Altman, D.G.; The PRISMA Group. Preferred Reporting Items for Systematic Reviews and Meta-Analyses: The PRISMA Statement. Ann. Intern. Med. 2009, 151, 264-269.

10. Higgins, J.P.T.; Green, S. Cochrane Handbook for Systematic Reviews of Interventions; Wiley-Blackwell: Hoboken, NJ, USA, 2009.

11. The Nordic Cochrane Centre. Review Manager (RevMan) [Computer Program]; Version 5.3; The Nordic Cochrane Centre, The Cochrane Collaboration: Copenhagen, Denmark, 2014.

12. Zhao, L.; Zhang, F.; Ding, X.; Wu, G.; Lam, Y.Y.; Wang, X.; Fu, H.; Xue, X.; Lu, C.; Ma, J.; et al. Gut bacteria selectively promoted by dietary fibers alleviate type 2 diabetes. Science 2018, 359, 1151-1156, doi:10.1126/science.aao5774.

13. Gurung, M.; Li, Z.; You, H.; Rodrigues, R.; Jump, D.B.; Morgun, A.; Shulzhenko, N. Role of gut microbiota in type 2 diabetes pathophysiology. EBioMedicine 2020, 51, 102590, doi:10.1016/j.ebiom.2019.11.051.

14. Reimer, R.A.; Wharton, S.; Green, T.J.; Manjoo, P.; Ramay, H.R.; Lyon, M.R.; Gahler, R.J.; \& Wood, S. Effect of a functional fibre supplement on glycemic control when added to a year-long medically supervised weight management program in adults with type 2 diabetes. Eur. J. Nutr. 2020, doi:10.1007/s00394-02002328-8.

Publisher's Note: MDPI stays neutral with regard to jurisdictional claims in published maps and institutional affiliations.

(C) 2020 by the authors. Submitted for possible open access publication under the terms and conditions of the Creative Commons Attribution (CC BY) license (http://creativecommons.org/licenses/by/4.0/). 